\title{
A propósito de "As duas parasitologias"
}

\author{
Mario B. Aragão*
}

Em complemento ao artigo apresentado nos " $\mathrm{Ca}$ dernos de Saúde Pública", Vol. 4, n: 4, outubro/dezembro de 1988, sob o título "As Duas Parasitologias", achamos da maior importância as referências bibliográficas utilizadas na preparação do mesmo.

\section{REFERÊNCIAS BIBLIOGRÁFICAS}

1. ARAGĀO, M. B. - Ecologia, evolução e saúde pública. Cad. Saúde publ., Rio de Janeiro, 2: $1-6$ (1981).

2. BAER, J. G. - Animal parasites. Translated by K. Lyons. Weiden feld and Nicolson, London (1971).

3. PRICE, P. W. - Insect ecology, 2nd ed. John Wiley \& Sons, New York (1984).

4. JARED, C. e FURTADO, M. F. - As serpentes. Ciência Hoje, Rio de Janeiro, 7: 26-3 (1988).

5. DOGIEL, V. A. - General parasitology. Translated by Z. Kabata, Oliver \& Boyd, Edinburgh and London (1964).

6. DEBACH, P. - Lucha biologica contra los enemigos de las plantas. Version española de M. A. Varela y C. S. Alvarez. Ediciones Mundi-Prensa, Madrid (1977).

7. PAVAN, C. - O exemplo da ecologia. Rev. Bras. Tecnol., Brasilia, 19: 40-41 (1988).

8. KREBS, C. J. - Ecology: The experimental analysis of distribution and abundance, 3rd ed. Harper \& Row Publishers, New York (1985).

9. BRUCE-CHWATT, L. J, e BRUCE-CHWATT, M. D. Malária in Mauritius - As dead as de dodo. Bull. $N$. Y. Acad. Med., 50:1069-1080 (1974).

10. LIMA, L. C. - Ruralizaçāo da Lutzomyia intermedia um provável caso de pré-adaptação. Rev. Saúde publ., São Paulo, 20: 102-104 (1986).

11. DEANE, M. P., LENZI, H. L. e JANSEN, A. M. Trypanosoma cruzi, vertebrate and invertebrate cycles in the same mamal host, the opossum $D$. marsupialis. Mem. Inst . Oswaldo Cruz, 79: 513-515 (1984).

12. SCHMIDT, G. D. e ROBERTS, L. S. - Foundations of parasitology, 2nd ed. The C. V. Mosby Company, St. Louis (1981).

13. PESSÔA, S. B. e MARTINS, A. V. - Pessôa patasitologia médica, 10: ed. Guanabara Koogan, Rio de Janeiro (1977).

14. YOUNG, M. D. - Monkeys and malaria. In: G. H. Bourne (ed.) Nonhuman primates and medical research. Academic Press, New York (1973).

15. DAMIAN, R. T. - Molecular mimicry in biological adaptation. In: B. B. Nickol (ed.) Host-parasite interfacies. Academic Press, New York (1979).

16. CAMER ON, T. W. M. - Parasites and parasitism. Methuen \& Co. Ltd., London (1956).

17. WHO Expert Commitee - Public health significance of intestinal parasitic infections. Bull. Wld Hlth Org., 65: 575-588 (1987).

* Pesquisador titular da Escola Nacional de Saúde Pública ENSP/FIOCRUZ
18. ARAGẢ , M. B. - Domiciliação de triatomíneos ou pré-adaptação à antropofilia e à ornitofilia? Rev. Saúde publ., S. Paulo, 17: 51-55 (1983).

Cadernos de Saúde Pública, RJ, 5 (2): 234-235, abr/jun, 1989 
19. ARAGĀO, M. B. - O comportamento dos anofelinos do subgênero Kerteszia, no sul do Brasil e o efeito do inseticida DDT. Mem. Inst. Oswaldo Cruz, 72: 147-172 (1974).

Agradecimentos: Aos Profs. Fernando Avila-Pires e Marcia Chame pela sugestão do tema e aos Drs. Adauto Araujo e Ulisses Confalonieri pelo empréstimo de bibliografia. 